\title{
Biomedical Teleacupuncture between China and Austria Using Heart Rate Variability_Part 2: Patients with Depression
}

\author{
Gerhard Litscher, ${ }^{1,2}$ Guangyu Cheng, ${ }^{3}$ Lu Wang, ${ }^{1,2}$ Weiping Cheng, ${ }^{2}$ \\ Hang Su, ${ }^{4}$ Qianqian Niu, ${ }^{2}$ Tianyu Zou, ${ }^{2}$ Yongyue Wang, ${ }^{2}$ Xiao Feng, ${ }^{2}$ \\ Ingrid Gaischek, ${ }^{1}$ Zemin Sheng, ${ }^{1,5}$ and Haixue Kuang ${ }^{2}$ \\ ${ }^{1}$ Stronach Research Unit for Complementary and Integrative Laser Medicine, Research Unit of Biomedical Engineering in Anesthesia
and Intensive Care Medicine, and TCM Research Center Graz, Medical University Graz, Auenbruggerplatz 29, 8036 Graz, Austria
${ }^{2}$ 2nd Neurology Department, Heilongjiang University of Chinese Medicine, No. 24 Heping Road, 150040 Harbin, China
${ }^{3}$ School of Chinese Medicine, Hong Kong Baptist University, 7 Baptist University Road, Kowloon Tong, Hong Kong
${ }^{4}$ Department of Geriatrics, Kunming Medical University, Kunming, 650031, China
${ }^{5}$ Chinese Medicine Department, Privatklinik Laßnitzhöhe, Miglitzpromenade 18, 8301 Laßnitzhöhe, Austria
}

Correspondence should be addressed to Gerhard Litscher, gerhard.litscher@medunigraz.at

Received 12 January 2012; Accepted 2 February 2012

Academic Editor: Xinyan Gao

Copyright (c) 2012 Gerhard Litscher et al. This is an open access article distributed under the Creative Commons Attribution License, which permits unrestricted use, distribution, and reproduction in any medium, provided the original work is properly cited.

\begin{abstract}
It has been shown in previous studies that the autonomic nervous system can be affected by acupuncture. Within this study, teleacupuncture between China and Austria is used for quantifying the effects of heart rate (HR) and heart rate variability (HRV) in 33 Chinese patients (27 females, 6 males; mean age \pm SD $49.5 \pm 13.1$ years; range 22-72 years) suffering from depression. Electrocardiographic signals before, during, and after acupuncture at the acupoint Baihui (GV20) were recorded in Harbin and analyzed in Graz using teleacupuncture. HRV data were analyzed in the time and frequency domain. Mean HR decreased significantly $(P<0.05)$ during and after acupuncture, whereas total HRV increased significantly after the third acupuncture stimulation period $(P<0.05)$ and also 5-10 minutes after $(P<0.05)$ acupuncture. The study shows that HRV could be a useful parameter for quantifying clinical effects of acupuncture on the autonomic nervous system.
\end{abstract}

\section{Introduction}

A recent Cochrane review identified 30 randomized controlled trials (RCTs) that evaluated manual acupuncture, electroacupuncture, or laser acupuncture in 2812 patients with major depressive disorder [1]. In this study, no consistent benefit was noted with any form of acupuncture [1]. However, our research group found acute stimulation effects on neurovegetative parameters like heart rate (HR) and heart rate variability (HRV) in patients with depression [2] and insomnia [3] and poststroke patients [4].

An innovative concept of the current teleacupuncture technology has been implemented at the Traditional Chinese Medicine (TCM) Research Center Graz in Austria (http:// litscher.info/ and http://tcm-graz.at/) in 2010 in cooperation with different institutions in China over a distance of several thousands of kilometres [5-7].

This paper describes the second results from teleacupuncture measurements in patients with depression using computer-based HRV recordings before, during, and after acupuncture under standardized clinical conditions in China. The first study in patients with depression was performed using the acupuncture point Jianshi (PC5) [2] and the present study the acupoint Baihui (GV20). All analyses were performed in Graz, Austria [5].

\section{Subjects and Methods}

2.1. Patients. Thirty-three patients (27 females, 6 males; mean age \pm SD $49.5 \pm 13.1$ years; range $22-72$ years) 
suffering from depression (Chinese diagnosis "Yu Zheng") and therefore receiving acupuncture treatment were investigated at the Heilongjiang University in Harbin. Similar to our first study the clinical evaluation of the patients was performed immediately before HRV data recording using three main scales: the Hamilton rating scale for depression (HRSD) [8], the Hamilton anxiety rating scale (HAM-A) [9], and the Athens insomnia scale (AIS) [10]. No patient was under the influence of centrally active medication. The study was approved by the ethic committee of the Heilongjiang University of Chinese Medicine (no. 2010HZYLL-030) and carried out in compliance with the Declaration of Helsinki. All patients gave oral informed consent.

\subsection{Biosignal Recording in Asia and Data Analysis in Europe.} The duration of RR intervals is measured during a special time period ( $5 \mathrm{~min}$ ), and on spectral analysis basis HRV is determined. Electrocardiographic (ECG) registration is performed using three adhesive electrodes (Skintact Premier F-55; Leonhard Lang GmbH, Innsbruck, Austria), which are applied to the chest.

The researchers in China used a medilog AR12 HRV (Huntleigh Healthcare, Cardiff, UK) system from the TCM Research Center at the Medical University in Graz for the joint investigations. This system has a sampling rate of $4096 \mathrm{~Hz}$ and can therefore detect $\mathrm{R}$ waves extremely accurately [11]. The raw data are stored digitally on a CompactFlash (CF) $32 \mathrm{MB}$ memory card. After removing the card from the portable system, the data were read by a card reader connected with a standard computer in China and then transferred to the TCM Research Center Graz via internet. With a new software [5-7] the biosignals were analyzed and HRV was displayed in a way to help to judge the function of the autonomic nervous system. Viewing this innovative kind of analysis helps to show how well the human body reacts to sport, stress, recovery, and also acupuncture $[2-7,12]$.

Similar to a previous studies [2-4], mean HR, total HRV, and the LF (low frequency)/HF (high frequency) ratio of HRV were chosen as evaluation parameters, as such being recommended by the Task Force of the European Society of Cardiology and the North American Society of Pacing and Electrophysiology [13].

2.3. Clinical Acupuncture and Procedure. All 33 patients received manual needle acupuncture at the acupoint Baihui (GV 20) on the head (Figure 1). Baihui is located 5 cun directly above the midpoint of the anterior hairline, at the midpoint of the line connecting the apexes of both ears. Its use is indicated, for example, in neurological diseases like depression, headache, dizziness, epilepsy, and mania [14]. Sterile single-use needles $(0.30 \times 25 \mathrm{~mm}$; Huan Qiu, Suzhou, China) were used. Needling was performed horizontally (angle $15^{\circ}$, depth about 1 cun), and the needle was stimulated clockwise and counterclockwise for 15 seconds each, with six rotations per second, resulting in 90 rotations per stimulation. Stimulation was done immediately after

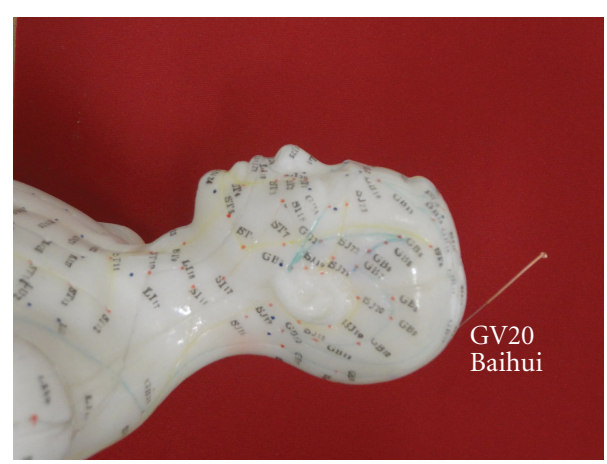

FIGURE 1: Acupuncture at the acupoint Baihui (GV20).

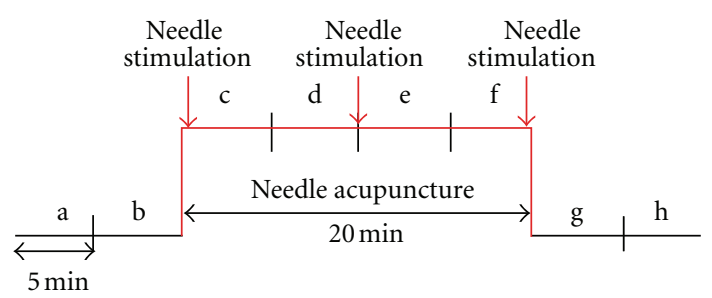

FIgURE 2: Measurement procedure. The data before (measurement phases a, b), during (c-f), and after ( $g$, h) manual needle acupuncture stimulation at the acupoint Baihui (comp. Figure 1) were measured and statistically analyzed.

inserting the needle, 10 minutes later and before removing the needle (cf. Figure 2 and [2]).

2.4. Statistical Analysis. Data of the 33 patients from the 2nd Neurological Department from the Heilongjiang University of Chinese Medicine in Harbin were analyzed using SigmaPlot 11.0 software (Systat Software Inc., Chicago, USA). Graphical presentation of results uses box plot illustrations. Testing was performed with Friedman repeated measures ANOVA on ranks and Tukey test. The criterion for significance was $P<0.05$.

\section{Results}

Figure 3 shows the results of mean HR from the ECG recordings before, during, and after acupuncture of the 33 patients with depression. There was a significant decrease in HR during the second half of the acupuncture phase and after acupuncture $(P<0.05)$.

In contrast to this decrease in HR, total HRV increased significantly $(P<0.05)$ only after finishing the third and last needle stimulation (Figure 4, phase g). This increase was still present at the end of the measurement procedure in comparison to the second phase (Figure 4, phase h; $P<$ 0.05). It is interesting that between the stimulation phases total HRV was lowered again, with the median continually increasing with respect to the previous nonstimulation phase. 


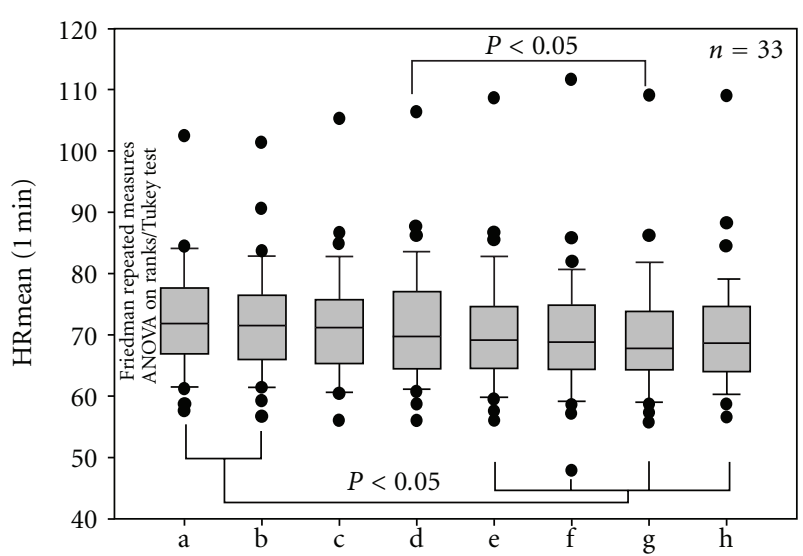

Figure 3: Mean heart rate. Box plot illustration in 33 patients with depression before $(a, b)$, during $(c-f)$, and after $(g, h)$ needle acupuncture. Significant changes $(P<0.05)$ were found in the phases $\mathrm{e}-\mathrm{h}$, each compared to baseline values $\mathrm{a}, \mathrm{b}$, respectively. The horizontal line in the box gives the position of the median. The end of the box defines the 25th and 75th percentile; the error bars mark the 10th and 90th percentile.

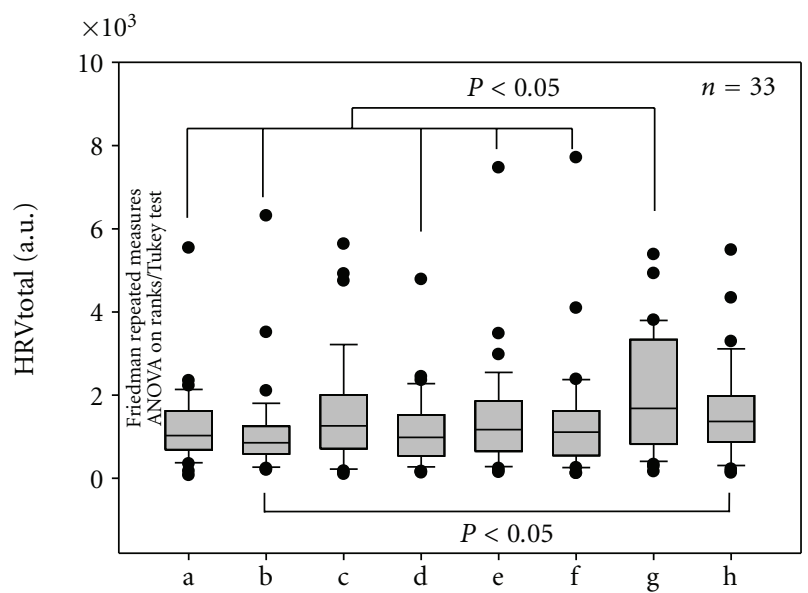

Figure 4: Total heart rate variability. Graphical box plot presentation of changes immediately after (g) and 5-10 min after (h) acupuncture. Note the increase in total HRV after each manual needle stimulation (c, e, g). For further explanations compare Figures 2 and 3.

Insignificant changes were found in the LF/HF ratio during acupuncture and can be seen in Figure 5.

The results of the different scales as described in Section 2 showed the following mean \pm SD values: HRSD $20.3 \pm 4.1$; HAM-A $19.4 \pm 4.4$; AIS $12.7 \pm 4.8$.

\section{Discussion}

Depression is one of the most prevalent and fastest-growing diseases in both western and eastern worlds. New-generation antidepressants appear more effective than older drugs; however, many drugs have side effects that can affect compliance and morbidity [1]. From the point of view of TCM the main syndromes of depression are qi stagnation, and blood

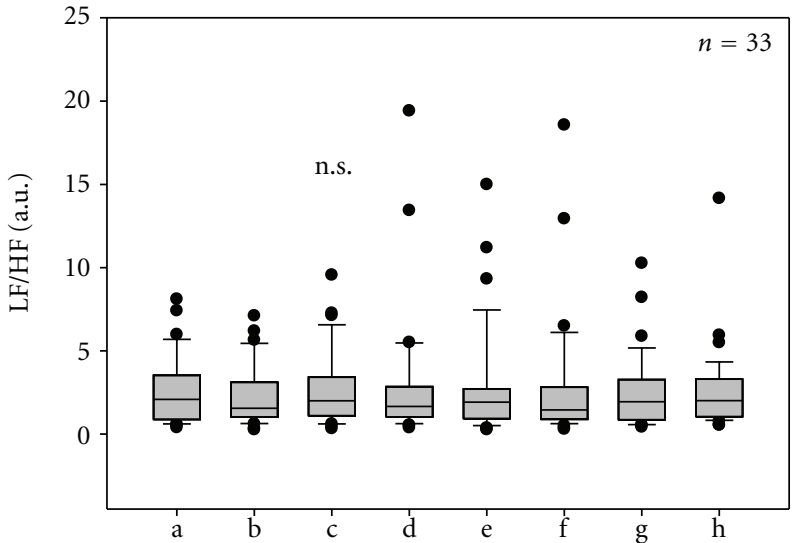

FIGURE 5: LF (low frequency)/HF (high frequency) ratio during acupuncture treatment in the 33 patients. For further explanations see Figure 3.

stasis, liver qi depression and transformation of fire due to qi stagnation [15]. In China, there are several preclinical and clinical studies using Chinese herbal medicine, which are the basis for design of new therapeutic programmes for treatment of depression [15]. In addition, acupuncture is also used in several evidence-based studies concerning this topic of research.

Although there are a great number of referenced publications (see Section 1 and http://www.pubmed.gov), there are only seven articles (including our first study on the topic [2]) concerning depression, acupuncture, and HRV at the moment (January 2012). These publications should be discussed in context with the results of this study in the following [2]: in 2001, Callahan [16] stated that HRV has been shown to be a strong predictor of mortality and is adversely affected by problems such as anxiety and depression. Pignotti and Steinberg [17] demonstrated that a lowering of subjective units of distress was in most cases also related to an improvement in HRV. In the third paper in 2001, Sakai et al. [18] included HRV in a general concept of behavioural health services, and the authors reported HRV as a useful parameter. In 2003, Agelink et al. [19] also undertook a study to evaluate the effects of needle acupuncture on cardiac autonomic nervous system function in patients with minor depression or anxiety disorders. In contrast to our 33 patients, the 36 patients from that group were randomly distributed into a verum acupuncture group and a placebo group. Similar to our investigations, 5minute intervals of ECG were analyzed and the acupuncture group also showed a significant decrease of the mean resting heart rate, 5 and 15 minutes after needle application (cf. Figure 3). In the study by Agelink et al. [19], this effect was only significant in verum acupuncture in patients with minor depression or anxiety. Therefore, a relative increase of cardiovagal modulation of heart rate and physiological regulatory effects due to acupuncture stimulation could be detected in the present study, which confirms the results of other authors [19], although the acupuncture schemes were 
different (He.7 Shenmen and PC7 Neiguan [19] versus PC5 Jianshi [2] and GV20 Baihui (this study)).

In a further publication, Yun et al. [20] described in 2005 the dynamic range of biologic functions. They stated that reduced variation of physical exertion, environmental stressors, and thermal gradients that characterize modern life styles may reduce the autonomic dynamic range resulting in lowered HRV and a myriad of systemic dysfunctions. Acupuncture may operate through increasing autonomic variability.

As already mentioned in the previous part (part 1) of this study [4], a systematic clinical review on acupuncture and HRV was published by Lee et al. in 2010 [21], which searched the literature using 14 data bases. Twelve RCTs met all inclusion criteria. Five RCTs found significant differences in HRV between patients treated with acupuncture and those treated with sham acupuncture (controls). The majority of the other RCTs showed inconsistent results [21]. The authors stated that more rigorous research appears to be warranted. The number, size, and quality of the RCTs that are available are too low to draw firm conclusions [21]. Another review article concerning the topic of HRV and acupuncture was published by our research group already in 2007 [12]. In this paper, it could be demonstrated that in special syndromes like fatigue and stress one can counteract the aging process using different preventive methods like acupuncture [12]. This was demonstrated in recent investigations concerning patients with burn-out syndrome as performed in a further teleacupuncture study between Beijing and Graz [5, 22].

The following conclusions can be drawn from the present clinical teleacupuncture study in patients with depression.

(1) The stimulation at the acupoint Baihui (GV20) significantly decreased HR in depression patients.

(2) Total HRV increased significantly during and after acupuncture stimulation at Baihui.

(3) We have shown that teleacupuncture at the acupoint Baihui in patients with depression shows similar effects in neurovegetative parameters like acupoint stimulation of Jianshi (PC5). In both studies ([2] and present study) the same technique in different patients but with the same disease was used.

\section{Authors' Contribution}

G. Litscher, G. Cheng and L. Wang contributed equally to this study.

\section{Acknowledgments}

The scientific investigations were supported by funds from the Oesterreichische Nationalbank (Anniversary Fund, Project no. 13463), the Stronach Medical Group (Chairman Frank Stronach), and the German Academy of Acupuncture (DAA; President Gerhard Opitz). The measurements were performed within the research areas of "Sustainable Health Research" and "Neuroscience" at the Medical University of
Graz. Professor G. Litscher and Dr. L. Wang are Visiting Professors at the Heilongjiang University of Chinese Medicine in Harbin, China. Professor G. Litscher is also Visiting Professor at the Institute of Acupuncture and Moxibustion at the China Academy of Chinese Medical Sciences in Beijing.

\section{References}

[1] R. Nahas and O. Sheikh, "Complementary and alternative medicine for the treatment of major depressive disorder," Canadian Family Physician, vol. 57, no. 6, pp. 659-663, 2011.

[2] L. Wang, G. Y. Cheng, Z. M. Sheng et al., "Clinical teleacupuncture between China and Austria using heart rate variability in patients with depression," Chinese Medicine, vol. 2, pp. 71-76, 2011.

[3] G. Litscher, G. Y. Cheng, W. P. Cheng et al., "Sino-European transcontinental basic and clinical high-tech acupuncture studies, part 2: acute stimulation effects on heart rate and its variability in patients with insomnia," Evidence-Based Complementary and Alternative Medicine, vol. 2012, Article ID 916085, 5 pages, 2012.

[4] L. Wang, J. Valentini, K. Sugimoto et al., "Biomedical teleacupuncture between China and Austria using heart rate variability, part 1: poststroke patients," Evidence-Based Complementary and Alternative Medicine, vol. 2011, Article ID 782489, 5 pages, 2011.

[5] G. Litscher, "Transcontinental and translational high-tech acupuncture research using computer-based heart rate and "Fire of Life" Heart Rate Variability Analysis," Journal of Acupuncture and Meridian Studies, vol. 3, no. 3, pp. 156-164, 2010.

[6] G. Litscher, "Transcontinental acupuncture research using teleacupuncture," Promed Komplementär, vol. 2, no. 3, pp. 8$11,2009$.

[7] G. Litscher, "Teleacupuncture: a new approach for transcontinental long-distance cooperation between Graz, Austria and Beijing, China (7,650 km)," Medical Acupuncture, vol. 21, no. 3, pp. 223-224, 2009.

[8] M. Hamilton, "A rating scale for depression," Journal of Neurology, Neurosurgery, and Psychiatry, vol. 23, pp. 56-62, 1960.

[9] M. Hamilton, "The assessment of anxiety states by rating," The British Journal of Medical Psychology, vol. 32, no. 1, pp. 50-55, 1959.

[10] C. R. Soldatos, D. G. Dikeos, and T. J. Paparrigopoulos, "Athens Insomnia Scale: Validation of an instrument based on ICD-10 criteria," Journal of Psychosomatic Research, vol. 48, no. 6, pp. 555-560, 2000.

[11] G. Litscher and D. Litscher, "Fire of Life' analysis of heart rate variability during alpine skiing in Austria," North American Journal of Medical Sciences, vol. 2, no. 6, pp. 258-262, 2010.

[12] G. Litscher, "Bioengineering assessment of acupuncture, Part 7: Heart rate variability," Critical Reviews in Biomedical Engineering, vol. 35, no. 3-4, pp. 183-195, 2007.

[13] M. Malik, A. J. Camm, J. T. Bigger Jr. et al., "Heart rate variability. Standards of measurement, physiological interpretation, and clinical use," European Heart Journal, vol. 17, no. 3, pp. 354-381, 1996.

[14] J. Yan, Skills with Illustrations of Chinese Acupuncture and Moxibustion, Hunan Science \& Technology Press, Changsha, China, 2006.

[15] H. Han, L. M. Wu, W. M. Yang et al., "Characteristics of traditional Chinese medicine syndromes in post-stroke 
depression," Journal of Chinese Integrative Medicine, vol. 8, no. 5, pp. 427-431, 2010.

[16] R. J. Callahan, "The impact of thought field therapy on heart rate variability," Journal of Clinical Psychology, vol. 57, no. 10, pp. 1153-1170, 2001.

[17] M. Pignotti and M. Steinberg, "Heart rate variability as an outcome measure for Thought Field Therapy in clinical practice," Journal of Clinical Psychology, vol. 57, no. 10, pp. 1193-1206, 2001.

[18] C. Sakai, D. Paperny, M. Mathews et al., "Thought Field Therapy clinical applications: utilization in an HMO in behavioral medicine and behavioral health services," Journal of Clinical Psychology, vol. 57, no. 10, pp. 1215-1227, 2001.

[19] M. W. Agelink, D. Sanner, H. Eich et al., "Does acupuncture influence the cardiac autonomic nervous system in patients with minor depression or anxiety disorders?" Fortschritte der Neurologie Psychiatrie, vol. 71, no. 3, pp. 141-149, 2003.

[20] A. J. Yun, K. A. Bazar, A. Gerber, P. Y. Lee, and S. M. Daniel, "The dynamic range of biologic functions and variation of many environmental cues may be declining in the modern age: implications for diseases and therapeutics," Medical Hypotheses, vol. 65, no. 1, pp. 173-178, 2005.

[21] S. Lee, M. S. Lee, J. Y. Choi, S. W. Lee, S. Y. Jeong, and E. Ernst, "Acupuncture and heart rate variability: a systematic review," Autonomic Neuroscience, vol. 155, no. 1-2, pp. 5-13, 2010.

[22] G. Litscher, "Translational research in acupuncture-teleacupuncture bridges science and practice," Health, vol. 2, no. 1, pp. 16-19, 2010. 


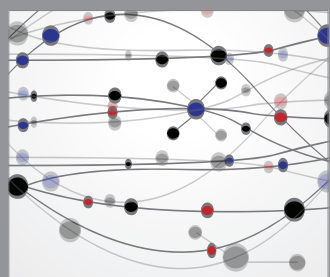

The Scientific World Journal
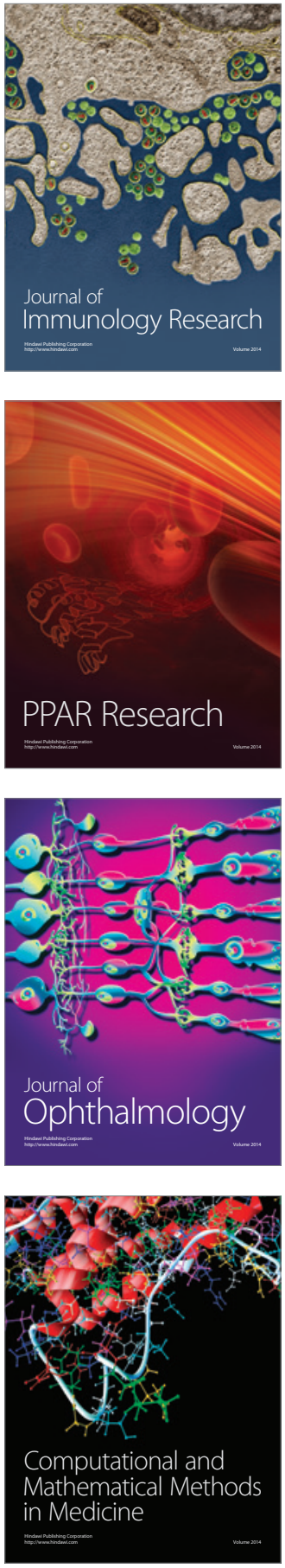

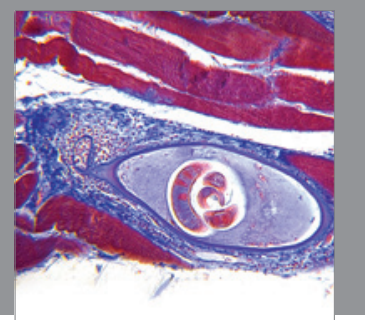

Gastroenterology

Research and Practice
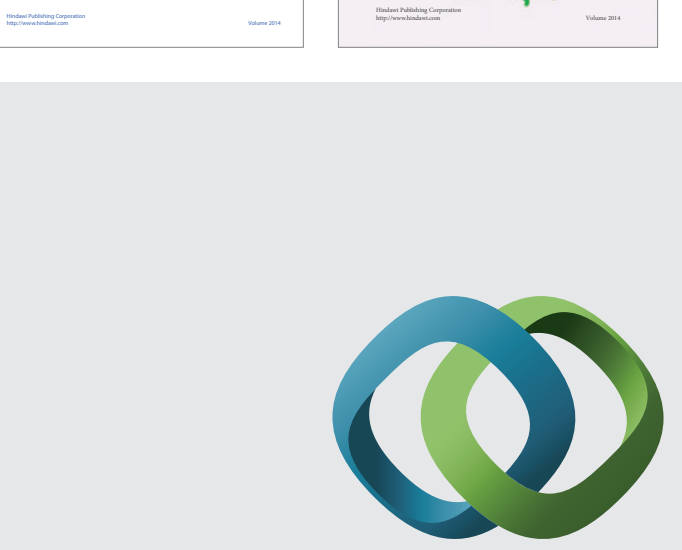

\section{Hindawi}

Submit your manuscripts at

http://www.hindawi.com
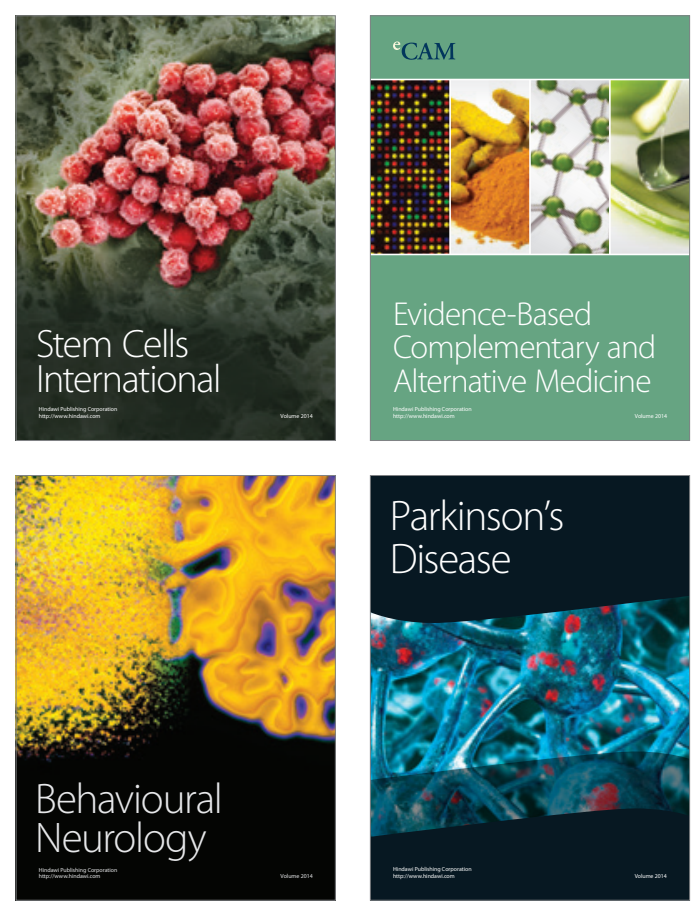

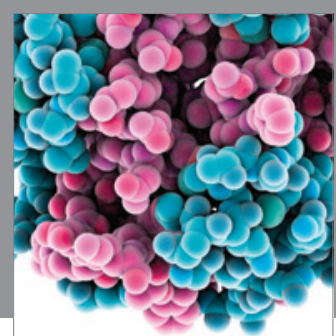

Journal of
Diabetes Research

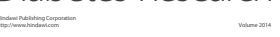

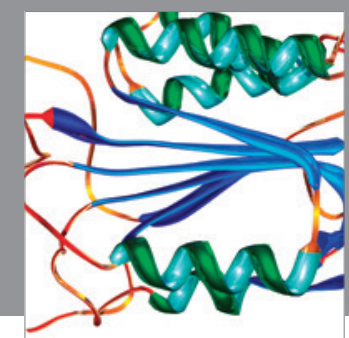

Disease Markers
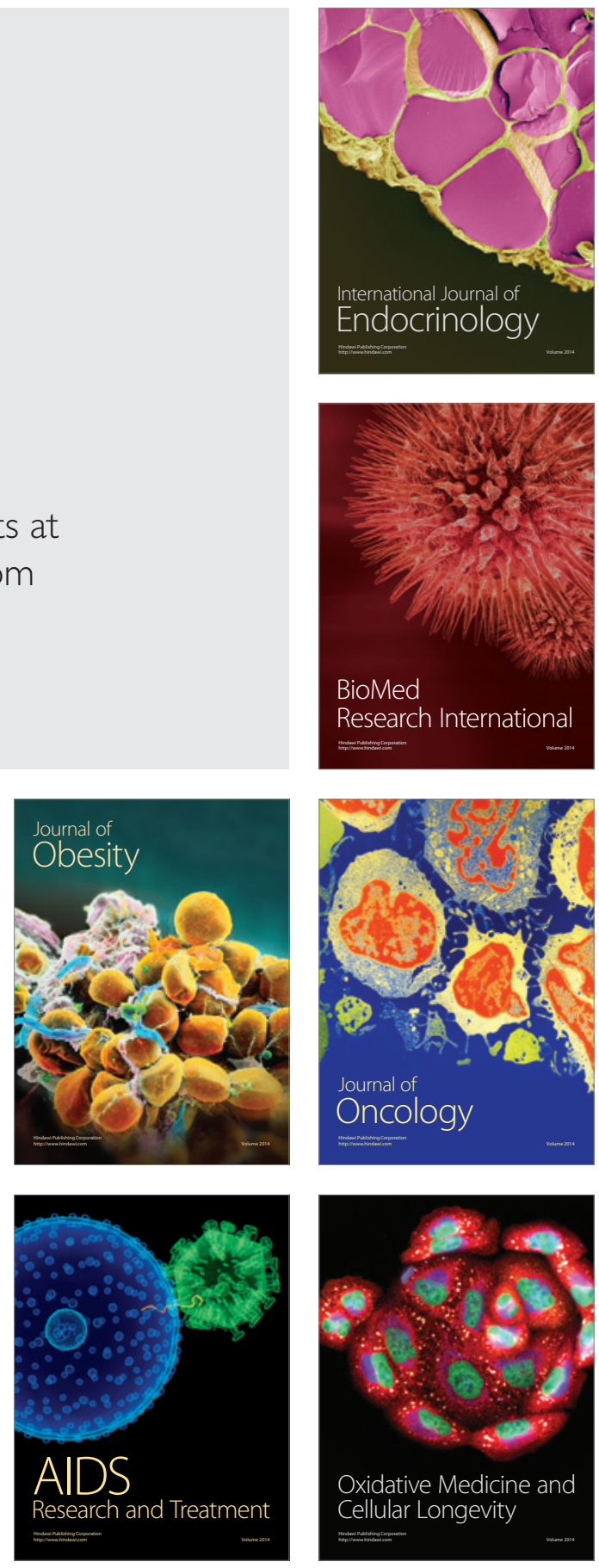